\title{
The Effects of Quinacrine, Proglumide, and Pentoxifylline on Seizure Activity, Cognitive Deficit, and Oxidative Stress in Rat Lithium-Pilocarpine Model of Status Epilepticus
}

\author{
Mohammad Ahmad, ${ }^{1}$ Gasem M. Abu-Taweel, ${ }^{2}$ \\ Ahmad E. Aboshaiqah, ${ }^{3}$ and Jamaan S. Ajarem ${ }^{4}$ \\ ${ }^{1}$ Department of Medical Surgical Nursing, College of Nursing, King Saud University, P.O. Box 642, Riyadh 11421, Saudi Arabia \\ ${ }^{2}$ Department of Basic Sciences, College of Education, Dammam University, P.O. Box 2375, Dammam 31451, Saudi Arabia \\ ${ }^{3}$ Department of Nursing Administration and Education, College of Nursing, King Saud University, P.O. Box 642, \\ Riyadh 11421, Saudi Arabia \\ ${ }^{4}$ Department of Zoology, College of Science, King Saud University, P.O. Box 2455, Riyadh 11451, Saudi Arabia
}

Correspondence should be addressed to Mohammad Ahmad; mbadshah@ksu.edu.sa

Received 26 July 2014; Revised 5 October 2014; Accepted 19 October 2014; Published 16 November 2014

Academic Editor: Vladimir Jakovljevic

\begin{abstract}
Copyright (C) 2014 Mohammad Ahmad et al. This is an open access article distributed under the Creative Commons Attribution License, which permits unrestricted use, distribution, and reproduction in any medium, provided the original work is properly cited.

The present data indicate that status epilepticus (SE) induced in adult rats is associated with cognitive dysfunctions and cerebral oxidative stress (OS). This has been demonstrated using lithium-pilocarpine (Li-Pc) model of SE. OS occurring in hippocampus and striatum of mature brain following SE is apparently due to both the increased free radicals production and the limited antioxidant defense. Pronounced alterations were noticed in the enzymatic, glutathione-S transferase (GST), catalase (CAT), and superoxide dismutase (SOD), as well as in the nonenzymatic; thiobarbituric acid (TBARS) and reduced glutathione (GST), indices of OS in the hippocampus and striatum of SE induced animals. Quinacrine (Qcn), proglumide (Pgm), and pentoxifylline (Ptx) administered to animals before inducing SE, were significantly effective in ameliorating the seizure activities, cognitive dysfunctions, and cerebral OS. The findings suggest that all the drugs were effective in the order of Ptx $<\mathrm{Pgm}<\mathrm{Qcn}$ indicating that these drugs are potentially antiepileptic as well as antioxidant; however, further studies are needed to establish this fact. It can be assumed that these antiepileptic substances with antioxidant properties combined with conventional therapies might provide a beneficial effect in treatment of epilepsy through ameliorating the cerebral OS.
\end{abstract}

\section{Introduction}

Status epilepticus (SE) is an emergency neurological condition where recurrent generalized seizures last for more than 30 minutes and if not controlled neuronal injury occurs $[1,2]$. Besides neurobehavioral deficits, SE is preferentially associated with a wide range of neurochemical imbalance in some areas of the brain [3-6]. Such neuronal hyperactivity and/or excitotoxicity have been associated with excessive generation of free radicals $[7,8]$, particularly in the brain, which contains large quantities of oxidizable lipids and metals, and moreover, the brain has fewer mechanisms of antioxidation than other tissues [9]. Oxidative stress, which is generally defined as the overproduction of free radicals, can dramatically alter neuronal function and has been linked to the pathogenesis of epilepsy $[8,10-12]$. There exist various endogenous antioxidant defense mechanisms, both enzymatic and nonenzymatic, which within certain limits can counteract increased ROS production.

Several neurochemical studies in animal models have revealed that oxidative stress-related seizures produce changes in antioxidant enzymatic activity and receptor binding $[8,13,14]$. The pathological process and underlying mechanisms involved in the oxidative stress during SE are still 
far from clear. There is ample evidence to suggest that brain tissues are highly vulnerable to the oxidative stress [15]. Reactive oxygen species (ROS) can dramatically affect the structure and function of neurons and recently Freitas et al. [15] suggested an evidence based role of ROS in the pathophysiology of SE. Importantly, epilepsy induced by pilocarpine in rodent models can provide information regarding oxidative stress-related epileptic activity [3, 4, 16-18]. Besides epileptic seizure activities, oxidative stress has also been related with cognitive impairment [19-22]. Furthermore, in such animal models, pharmacological approaches designed to reduce the oxidative stress afford significant neuroprotection, attesting to the critical role that oxidative stress plays in a diverse array of neurological diseases and disorders. Inhibition of the neurochemical pathways responsible for neuronal excitability and behavioral changes may lead to the development of new therapeutic agents for the treatment of SE.

The anticonvulsant effect of several agents having antioxidant property such as curcumin, vineatrol, transresveratrol, melatonin, adenosine, alpha lipoic acid, pentoxifylline, buspirone, aloe vera, and metformin has been demonstrated in various studies [2, 6, 23-28].

Proglumide (Pgm) is a known cholecystokinin (CCK) antagonist [29] and changes CCK level and receptor population of CCK has been associated with SE [30]. Quinacrine (Qcn) is a potent phospholipase A2 (PLA2) inhibitor [31] and has been shown to exert neuroprotective activity in ischemic brain [32]. PLA2 are a family of ubiquitous enzymes that degrade membrane phospholipids and produce a variety of lipid mediators to regulate neuronal functions [32]. PLA2 has also been shown to have a direct role in regulation of neurotransmitters and electrolytes in normal and reduced OS-mediated cellular toxicity. Pentoxifylline (Ptx) has also been shown to have neuroprotective effects against a host of neurobehavioral disorders including ischemic brain injury, neurotrauma, dementia, stroke, improved cognitive effect following hippocampal lesions, and ameliorated effects on lithium-pilocarpine induced SE in young rats [6, 33-35].

The pilocarpine- (Pc-) induced seizure in rodents is generally considered as one of the most suitable experimental models that has been frequently used to study the pathophysiology and management strategies of SE $[3,8,36]$. Due to high mortality of animals in pilocarpine (alone) model [37], a combination of lithium chloride and pilocarpine (LiPc) model is often preferred. Lithium (Li) potentiates the epileptogenic action of Pc but at the same time significantly reduces the mortality of animals $[38,39]$. The Li-Pc model of $\mathrm{SE}$ reproduces most clinical, temporal, and neuropathological features of SE $[3,6,40,41]$.

The present study was designed to study the comparative effects of Pgm, Qcn, and Ptx on epileptic seizure activities, cognitive tests, and oxidative stress in the brain regions of rats in which SE was induced by lithium-pilocarpine. Our prime objective was to assess if the oxidative stress observations in the SE animals can be of any help in identifying effective antiepileptic drugs.

\section{Materials and Methods}

2.1. Experimental Animals. The animals used in the present study were adult male Sprague Dawley rats (weighing 200$250 \mathrm{~g}, 2$ months old) and were housed under controlled conditions with 12 hours light-dark diurnal cycle at $22 \pm$ $1{ }^{\circ} \mathrm{C}$, with humidity at $50-60 \%$ and with free access to food and water except during experimental handlings. All study protocols and animal handling procedures were in accordance with and approved by the Research and Ethics Committee of King Saud University, Riyadh, Saudi Arabia. The prior permission was obtained from this committee for executing the experiments.

2.2. Induction of SE. Animals were randomly assigned into thirteen groups. The animals in groups 1, 2, and 3 served as controls and received saline, $\mathrm{Li}(3 \mathrm{mEq} / \mathrm{mL} / \mathrm{kg}$, i.p. $)$, and Pc alone $(20 \mathrm{mg} / \mathrm{mL} / \mathrm{kg}$, s.c. $)$, respectively. SE was induced in groups 4 to 13 by administering an aqueous (saline) solution of Li (BDH Laboratory Supplies, Poole, England, in a dose as in control), followed by ( $20 \mathrm{~h}$ later) Pc (Sigma Chemical Co., St. Louis, MO, USA, in the dose as used for control). Group 4 served as the experimental control of SE group and groups 5 to 13 served as the drug test groups. Qcn, Pgm, and Ptx (Sigma, USA) were dissolved in saline and were administered at doses of 5, 15, and $30 \mathrm{mg} / \mathrm{mL} / \mathrm{kg}$ i.p.; 250, 500 , and $750 \mathrm{mg} / \mathrm{mL} / \mathrm{kg}$ i.p.; and 20, 40, and $60 \mathrm{mg} / \mathrm{mL} / \mathrm{kg}$ i.p. (one hour before Pc injection), to groups 5 to 13, respectively. Groups receiving Qcn, Pgm, and Ptx alone in the highest dose $(30,750$, and $60 \mathrm{mg} / \mathrm{kg}$, resp.) served as the controls for the drugs used in this study. However, the results of these drug alone controls are not included in the results of this study, since these drugs administered alone in the animals did not show any abnormal behavioral activities and resembled the untreated controls. Furthermore, even all investigated parameters in this study for the drug alone groups were almost similar as the naïve control groups. Thus none of the results for the drug alone treated groups are included in the results of this study in order to avoid unnecessary confusion and crowding in the numbers of bars shown in the result figures. After Pc injections, the animals ( $n=10$ per group) were observed for a sequence of behavioral alterations, including peripheral cholinergic signs (PCS), stereotyped movements (STM), clonic movements of forelimbs, head bobbing, tremors, and seizures, which developed progressively within 1-2 h into SE [42]. For comparative observations on the drug effects, all seizure activities were presented as latency to develop seizure and SE. The seizure behavior consists of head bobbing with intermittent forelimb and hind limb clonus, hyperextension of tails, loss of posture, falling back, and myoclonic jerks, whereas SE is a condition where these recurrent generalized seizures last for more than 30 minutes in the animals. Mortality (if any) within $24 \mathrm{~h}$ was also recorded.

2.2.1. Morris Water-Maze Test. After induction of SE for seizure activities analyses, the animals ( $n=6$ to 8 per group) were subjected to cognitive test. Animals were allowed to 
acclimatize to the testing room for $2 \mathrm{~h}$ before testing. All tests were performed between 10:00 and 15:00 hours of the lighted phase.

The test has been extensively used to assess cognitive functions in a variety of epilepsy models [43, 44]. The rats were tested for visual-spatial memory using a water-maze [45]. The water-maze consisted of a galvanized white circular water tank (117 cm diameter, $55 \mathrm{~cm}$ height) filled with clear tap water $\left(26 \pm 1^{\circ} \mathrm{C}\right)$ to a depth of $30 \mathrm{~cm}$. A $10 \mathrm{~cm}$ diameter, stainless steel, white, escape platform was placed $1 \mathrm{~cm}$ below the water level and the water was made opaque by addition of 1 liter of milk, which prevented visualization of the platform. Four points on the rim of the tank were designated north $(\mathrm{N})$, south (S), east (E), and west (W), thus dividing the pool into four quadrants (NW, NE, SE, and SW).

On the first day, each rat was allowed to swim freely in the pool for $60 \mathrm{sec}$ without the platform present in the pool. This free swim enabled the rat to become habituated to the training environment. On days 2-5, rats were trained for 24 trials (six trials a day, with an intertrial interval of $30 \mathrm{sec}$ ) to locate and escape onto the submerged platform. At the start of each trial, the rat was held facing the perimeter of the water tank and dropped into the pool to ensure immersion. The latency from immersion into the pool to escape onto the hidden platform (maximum trial duration $120 \mathrm{sec}$ ) was recorded. On mounting the platform, each rat was given a $30 \mathrm{sec}$ intertrial interval for rest and for learning and memorizing the spatial cues to reach the platform for escape. The testing procedures used during the four days of locating the hidden platform provide a measure of hippocampaldependent spatial reference memory [46].

2.3. Biochemical Studies. Based on our pilot studies and literature survey $[5,47,48]$, the biochemical studies were undertaken $1 \mathrm{~h}$ after Li-Pc treatment and immediately after killing the rest of the animals by decapitation, their brains were dissected on ice, and cerebral areas (hippocampus and striatum) were removed and frozen in liquid nitrogen and stored at $-70^{\circ} \mathrm{C}$ for determination of some nonenzymatic and enzymatic oxidative stress indices.

\subsection{Determination of Nonenzymatic OS Indices}

2.4.1. Lipid Peroxides. Lipid peroxides (LP) in striatum and hippocampus were determined spectrophotometrically as thiobarbituric acid-reactive substances (TBARS) according to the method of Ohkawa et al. [49]. Tissue lipid peroxide levels were quantified using extinction coefficient of $1.56 \times$ $10^{5} \mathrm{~m}^{-1} \mathrm{~cm}^{-1}$ and expressed as nanomoles of TBARS formed per $g$ tissue weight. The results are expressed as nmol/g wet weight.

2.4.2. Glutathione. Reduced glutathione (GSH) level in striatum and hippocampus was measured enzymatically in the brain tissues by a slightly modified method [50]. The slope of the change in absorbance was used to quantitate total GSH by comparing the slope of the samples with a standard curve prepared with pure glutathione (Sigma). The specific activity is expressed into umol/g tissue weight.

\subsection{Determination of Enzymatic OS Indices}

2.5.1. Glutathione-S-Transferase. Glutathione S-transferase (GST) was estimated by the method of Habig et al. [51] by using 1-chloro-2,4-dinitrochlorobenzene (CDNB) as substrate at $340 \mathrm{~nm}$. The GST activity is expressed as U/g tissue weight.

2.5.2. Catalase. Catalase (CAT) activity was measured by the method of Aebi [52], by tracking the decomposition of hydrogen peroxide by measuring decrease in extinction of $\mathrm{H}_{2} \mathrm{O}_{2}$ at $240 \mathrm{~nm}$. The activity of CAT is expressed as rate constant of first order reaction $\mathrm{K}$ per gram tissue weight.

2.5.3. Superoxide Dismutase. Superoxide dismutase (SOD) activity was estimated by the method of Misra and Fridovich [53]. Activity is expressed as the amount of enzyme that inhibits the oxidation of epinephrine by $50 \%$ which is equal to U per gram tissue weight.

2.6. Statistical Analysis. The data were analyzed by Bartlett's test for equal variance and by Gaussian-shaped distribution for normality using the Kolmogorov-Smirnov goodness-offit test. As the data passed the normality test $(P>0.10)$, group means were compared with one-way ANOVA with post hoc testing using Tukey-Kramer Multiple Comparisons Test or Student-Newman-Keuls Multiple Comparisons Tests. Differences in seizure and SE incidences and mortality were tested by Student-Newman-Keuls Multiple Comparisons Tests. All results were expressed as means \pm SEM and the significance was defined as $P<0.05$ for all tests.

\section{Results}

3.1. Features of Li-Pc Induced SE. Within $5 \mathrm{~min}$ after injection of Pc, all animals started developing a gradual and significant change in behavior including PCS (miosis, piloerection, diarrohea, mild tremors, scratching, and salivation) and STM (sniffing, paw licking, and rearing) followed by seizures in $100 \%$ of the animals with a mean latency of $9.62 \pm 1.2 \mathrm{~min}$ to develop seizure (Table 1). The convulsive episode consisted of head bobbing with intermittent forelimb and hind limb clonus, hyperextension of tails, loss of posture, falling back, and myoclonic jerks building up to SE in $100 \%$ of animals. The mean latency to onset of SE was $23.86 \pm 1.54 \mathrm{~min}$ (Table 1 ), and on average, the SE lasted for more than one hour. A total of $10 \%$ mortality were observed over a period of $24 \mathrm{~h}$ following Pc injections (Table 1).

3.2. Effect of Drugs Pretreatment on Li-Pc Induced SE. Pgm, Qcn, and Ptx dose-dependently and significantly increased the latencies to seizure and SE and decreased the percentages of seizures and SE (Table 1) and also reduced the intensity and frequency of seizure, PCS, and STM episodes (not shown 


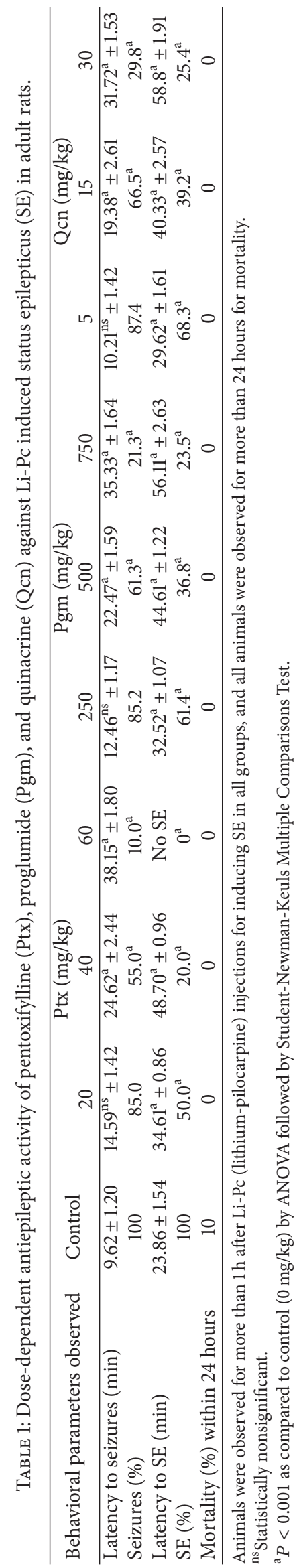



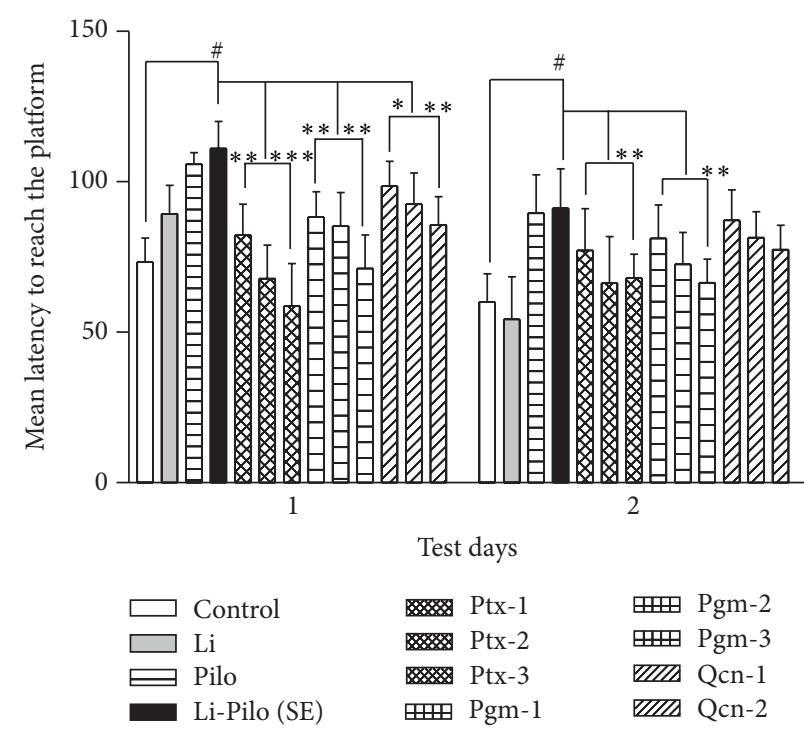

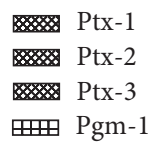

(a)
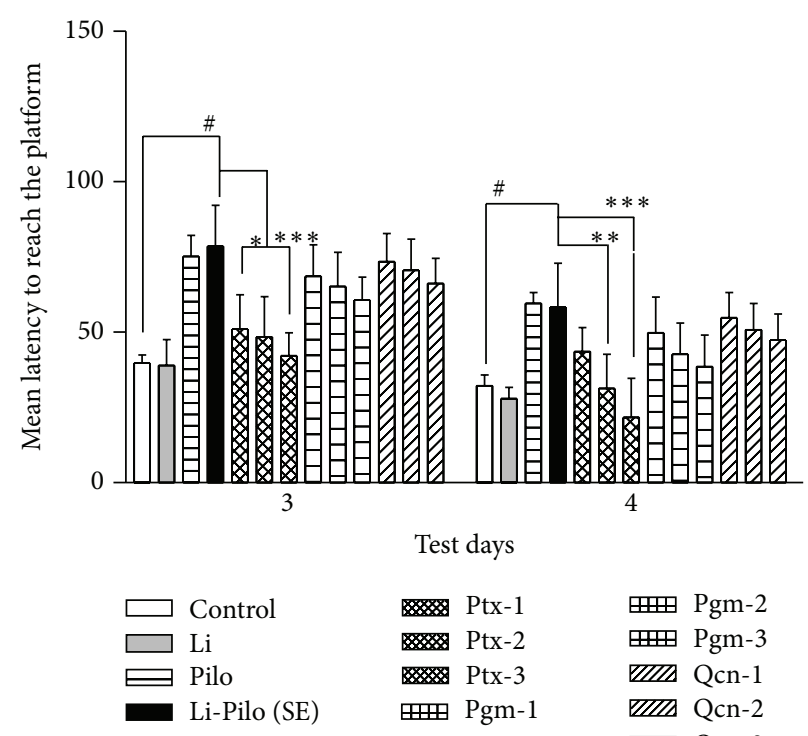

世 Pgm-2 世再 Pgm-3 IIIS Qcn-1 IIII Qcn-2 IIIS Qcn-3

(b)

FIGURE 1: Performance in water-maze of animals that experienced SE along with their controls as well as the SE groups that were pretreated with Ptx, Qcn, and Pgm. Mean latency to reach the hidden platform in seconds \pm SEM ( $y$-axis) on each testing day ( $x$-axis) shows that animals subjected to SE were slower in finding the platform (cognitive effect) than the controls on all four testing days. Treatment with Ptx, Qcn, and Pgm in varying doses was effective in improving the cognitive function in the order $\mathrm{Ptx}<\mathrm{Pgm}<\mathrm{Qcn}$ and dose-dependently. Li $=$ lithium chloride; Pilo = pilocarpine; SE = status epilepticus; doses of drugs in mg/kg body weight: Ptx-1, Ptx-2, and Ptx-3 = 20, 40, and 60; Pgm-1, Pgm2, and Pgm3 = 250, 500, and 750; and Qcn-1, Qcn-2, and Qcn-3 = 5, 15, and 30, respectively. \# represents significance as compared to control $(P<0.001)$, whereas $*, * *$ and $* * *$ show $P<0.01, P<0.05$, and $P<0.001$, respectively, as compared to SE group by one-way ANOVA.

in Table 1). The severity of SE was significantly and dosedependently reduced and latency to attain SE was increased in the drug treated animals. The drugs were effective in the order Ptx $>$ Pgm $>$ Qcn. The highest dose $(60 \mathrm{mg} / \mathrm{kg})$ of Ptx completely abolished SE (Table 1). Furthermore, no mortality was observed in the rats pretreated with all the three drugs, as compared to $10 \%$ mortality in the Li-Pc treated group (Table 1). The control groups that received Li or the drugs alone did not show any signs of seizure or SE.

3.3. Morris Water-Maze Test. Rats with Li-Pc treatment exhibited longer escape latencies to reach the platform as compared with control group $(P<0.01$; Figure 1$)$; however, all groups displayed gradual improvement in performance over the 4 days of testing (training) period. The number of successful animals to reach the platform was significantly higher in the drug pretreated groups as compared to Li-Pc (SE) group on all the four testing days but in the order Ptx $>$ Pgm $>$ Qcn $(P<0.001$; Figure 1$)$.

\subsection{Biochemical Studies}

\subsubsection{Nonenzymatic OS Indices}

TBARS in Hippocampus and Striatum. The lipid peroxidation level (TBARS) in the hippocampus and striatum was markedly $(P<0.001)$ increased after $1 \mathrm{~h}$ of Li-PC (SE) treatment as compared to the control group (Figure 2(a)).
Pretreatment with drugs significantly $(P<0.001)$ and dosedependently attenuated Li-Pc induced increase in TBARS in the hippocampus and striatum in the order Ptx $>$ Pgm $>$ Qcn (Figure 2(a)) as compared to Li-Pc (SE) group.

GSH in Hippocampus and Striatum. A highly significant $(P<0.001)$ depletion of hippocampal and striatal GSH was observed in Li-Pc (SE) group (Figure 2(b)). Pretreatment with drugs significantly and dose-dependently attenuated this depletion of GSH in the hippocampus and striatum in the order Ptx $>$ Pgm $>$ Qcn (Figure 2(b)) as compared to Li$\mathrm{Pc}$ (SE) group.

\subsubsection{Enzymatic OS Indices}

GST in Hippocampus and Striatum. A highly significant $(P<$ 0.001 ) depletion of hippocampal (Figure 3(a)) and striatal (Figure 3(b)) GST was also observed in Li-Pc (SE) group. Pretreatment with drugs significantly and dose-dependently attenuated this depletion of GST in the hippocampus and striatum in the order Ptx $>$ Pgm $>$ Qcn (Figures 3(a) and 3(b)) as compared to $\mathrm{Li}-\mathrm{Pc}$ (SE) group.

CAT in Hippocampus and Striatum. The CAT level in the hippocampus (Figure 3(c)) and striatum (Figure 3(e)) was markedly $(P<0.001)$ increased after $1 \mathrm{~h}$ of Li-PC (SE) treatment as compared to the control group (Figures 3(c) and $3(d))$. Pretreatment with drugs significantly $(P<0.001)$ and 

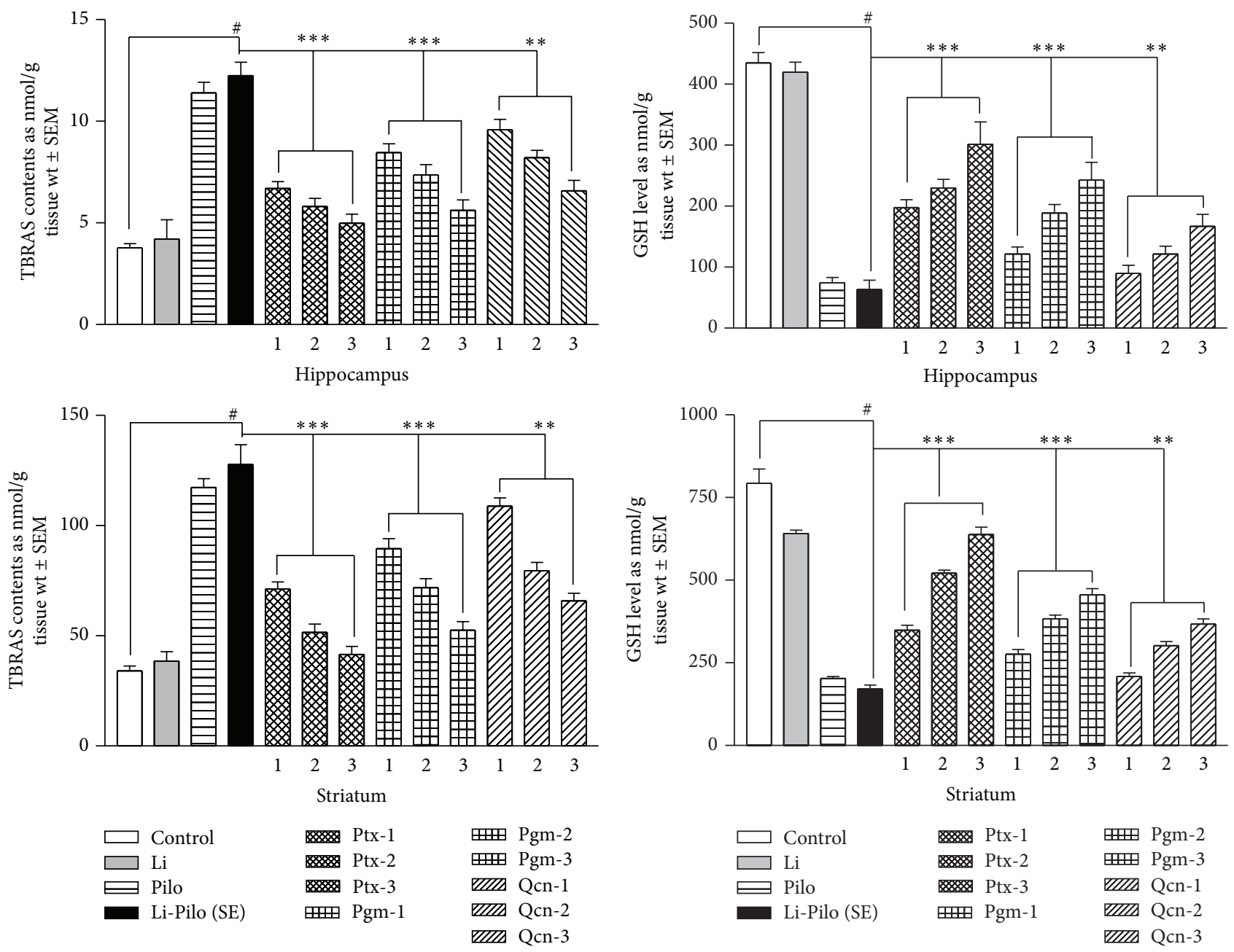

(a)

(b)

FIGURE 2: Comparative effect of Ptx, Pgm, and Qcn on the nonenzymatic oxidative stress indices like (a) lipid peroxidation content (TBARS) and (b) total glutathione level (GSH), in hippocampus and striatum of rats after Li-Pc induced SE. The comparative effects are in the order $\mathrm{Ptx}<\mathrm{Pgm}<\mathrm{Qcn}$ and dose-dependent. Abbreviations and statistical significance are the same as in Figure 1.

dose-dependently attenuated Li-Pc induced increase in CAT in the hippocampus and striatum in the order Ptx $>$ Pgm $>$ Qcn (Figures 3(c) and 3(d)) as compared to Li-Pc (SE) group.

SOD in Hippocampus and Striatum. The SOD level in the hippocampus (Figure 3(e)) and striatum (Figure 3(f)) was significantly $(P<0.001)$ decreased after $1 \mathrm{~h}$ of Li-PC (SE) treatment as compared to the control group. Pretreatment with drugs significantly $(P<0.001)$ and dose-dependently attenuated Li-Pc induced decrease in SOD in the hippocampus and striatum in the order Ptx $>$ Pgm $>$ Qcn (Figures 3(e) and $3(\mathrm{f})$ ) as compared to $\mathrm{Li}-\mathrm{Pc}$ (SE) group.

\section{Discussion}

The present findings indicate that Pc administration to rats pretreated with Li initiated cholinergic symptoms including miosis, piloerection, diarrhea, and mild tremors followed by seizures. SE developed between 20 to 30 minutes after Pc administration which consisted of head bobbing, intermittent forelimb and hind limb clonus, and hyperextension of tail and hind limb along with loss of posture. No mortality was found in any of the drug treated groups as compared to $10 \%$ mortality in the Li-Pc (SE) group. The present Morris water-maze results showed that rats with SE took longer time to reach escape platform, spent lesser time in the target quadrant, or completely failed to reach the platform clearly suggesting impaired visual-spatial memory and cognitive deficit. Cognitive dysfunctions were also evident from the fact that the animals swam along with the wall of the test tank and rarely tried to find the escape platform in the target quadrant. Impairment of learning and memory in rats with SE has been reported by several investigators $[6,54,55]$. The specific cause of motor and cognitive deterioration following SE is far from clear. However, according to recent reports neurochemical imbalance and alteration of neuronal structure following SE might be responsible for neurobehavioral changes [39, 47]. Cha et al. [56] reported that hippocampus receives dense 


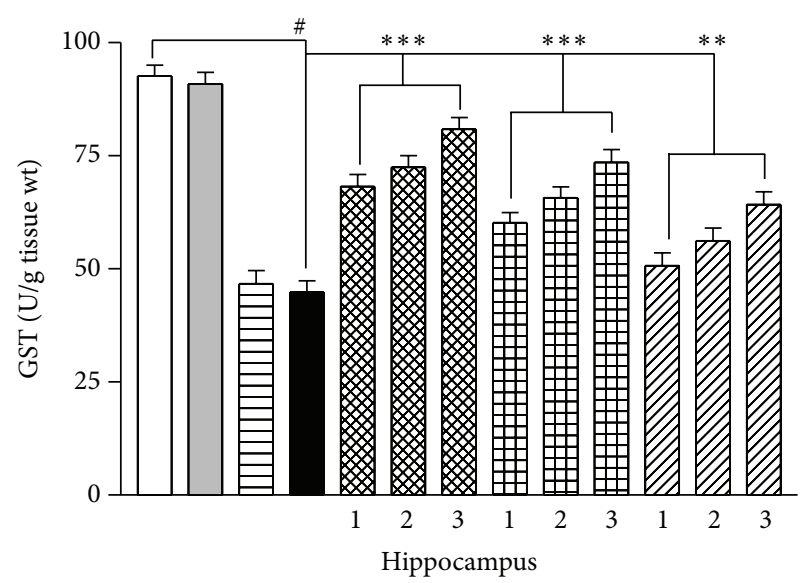

(a)

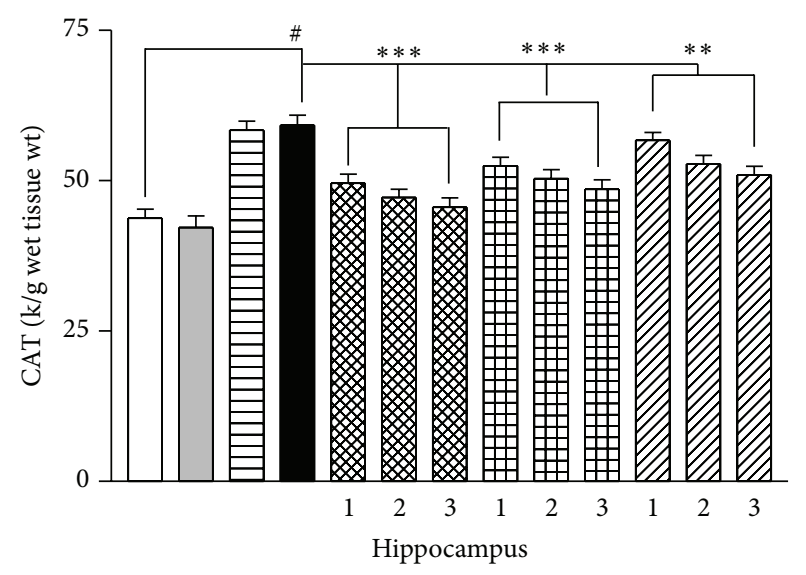

(c)
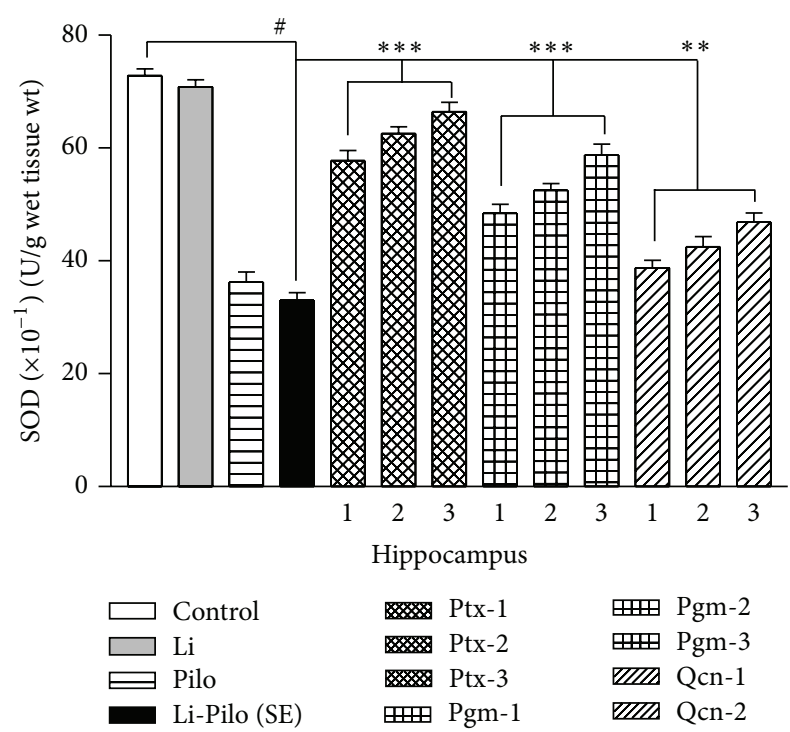

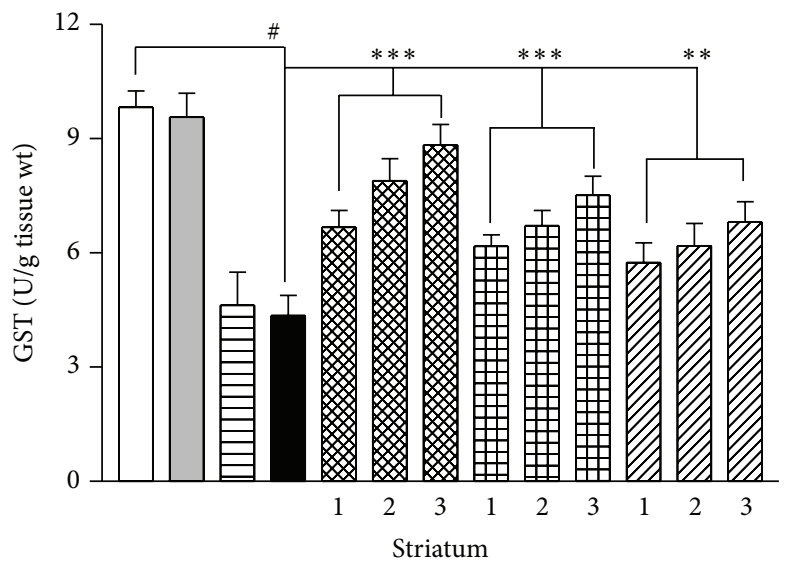

(b)

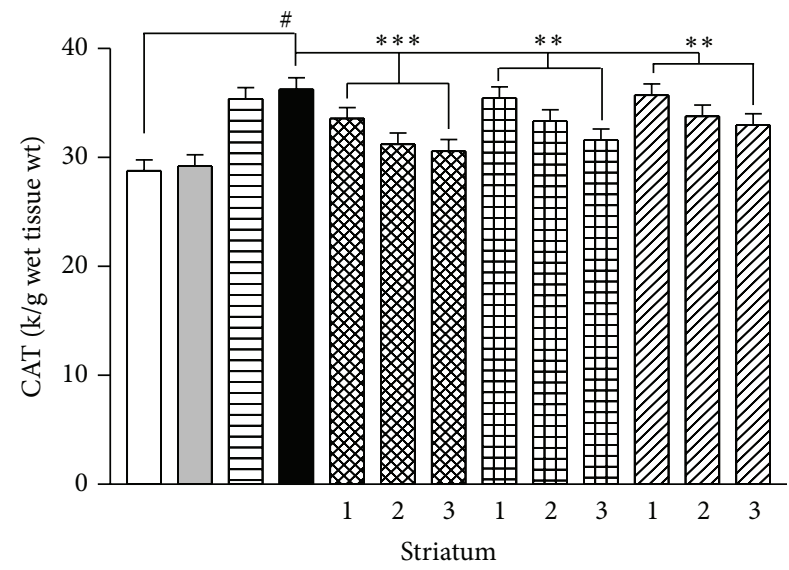

(d)
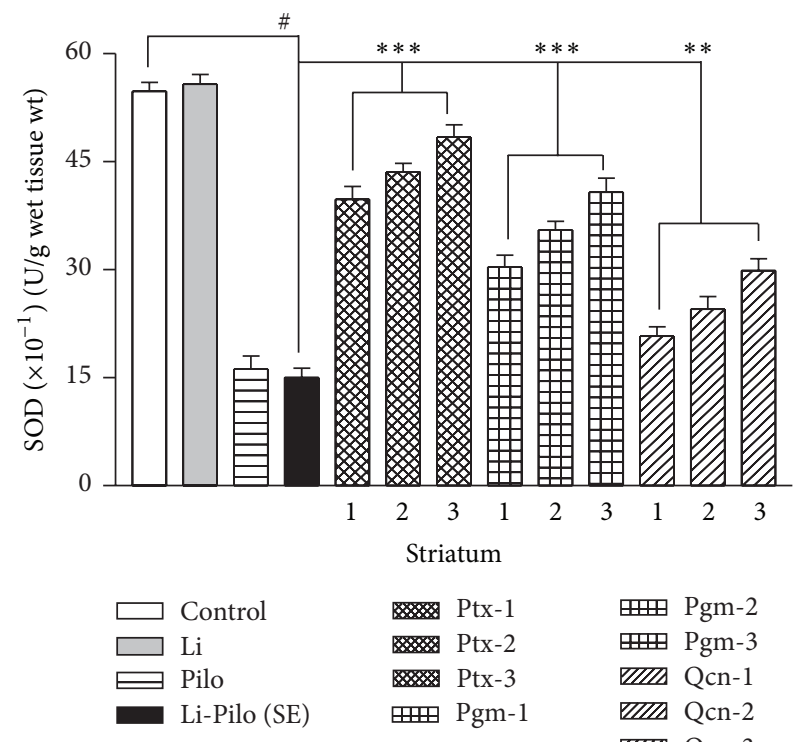

(e)
[या Qcn-3

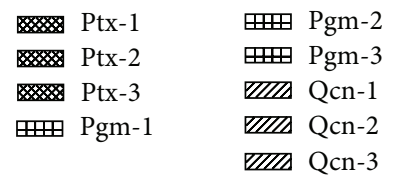

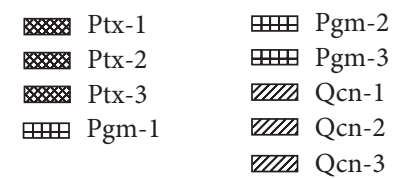

(f)

Figure 3: Comparative effect of Ptx, Pgm, and Qcn on the enzymatic oxidative stress indices like glutathione-S-transferase level (GST) in hippocampus (a) and striatum (b); catalase (CAT) activity in hippocampus (c) and striatum (d) and superoxide dismutase (SOD) activity in hippocampus (e) and striatum (f) of rats after Li-Pc induced SE. The comparative and dose-dependent effects are in the order Ptx $<$ Pgm $<$ Qcn. Abbreviations and statistical significance are the same as in Figure 1. 
cholinergic projections and overactivation of these afferents by Pc may directly produce neuronal hyperexcitation and trigger seizure activity accompanied by alterations in neuronal plasticity within the hippocampal circuitry causing impairment of learning and memory in rats $[44,57,58]$.

The results of the present study clearly showed the antiepileptic activity of all three drugs (Pgm, Qcn, and Ptx) against Li-Pc induced seizure, as revealed by highly significant decrease in frequency of epileptic episodes and increase in the latency to SE. The mortality was absent in all the three drug treated groups and the comparative efficacy of the tested drugs was in the order Ptx $>$ Pgm $>$ Qcn (Table 1). Furthermore, our study consistently demonstrated that pharmacological intervention using Pgm, Ptx, and Qcn significantly and dose-dependently attenuated SE induced impaired memory and the comparative efficacy of the tested drugs was in the same order Ptx $>$ Pgm $>$ Qcn throughout.

The present biochemical studies indicated a significant and dose-dependent increase in TBARS and CAT (Figures 2(a) and 2(b)) and decrease in GSH, GST, and SOD levels (Figures 3(a)-3(f)) in the hippocampus and striatum of the rats treated with $\mathrm{Li}-\mathrm{Pc}$ clearly suggesting a high level of enzymatic (GST, SOD, and CAT) and nonenzymatic (TBARS and GSH) oxidative stress in these brain regions. It is widely accepted that tissue injury is dependent not only on the nature of offending pathogen but also on the quality of host defense system including the type and levels of antioxidants. Oxidative stress is particularly facilitated in brain since the neuronal cells contain large quantities of oxidizable lipids and metals and the antioxidant defense mechanism in brain is relatively weaker as compared to other organs [8]. The drugs used in the present study significantly and dosedependently attenuated Li-Pc induced OS in hippocampus and striatum but in the order Ptx $>$ Pgm $>$ Qcn (Figures 2 and 3). Earlier, it has been reported [6] that Ptx may exert its pharmacological effects by several mechanisms including inhibition of phosphodiesterase enzymes (PDEs), leading to increases in cyclic adenosine monophosphate (cAMP), blockade of adenosine receptors, translocation of extracellular calcium, an inhibitory effect on inflammatory mechanism, and free radical scavenging activity. The role of cAMP in the etiopathology of epileptic seizures has been widely studied $[59,60]$. Besides its definite association with seizure activity, cAMP also plays a key role in biochemical regulation of the cognitive process of memory consolidation [61]. Earlier studies have also revealed that OS-related seizures produce changes in antioxidant enzyme activities $[6,8,13,14]$ and still other studies have related Pc-induced epileptic activity with the disturbance in OS activities $[3,4,16]$. Neuronal hyperexcitability and excessive production of free radicals have been implicated in the pathogenesis of a considerable range of neurological disorders, including epilepsy [62-64]. Lipid peroxidation in a tissue is an index of irreversible biological damage of the cell membrane phospholipid, which in turn leads to inhibition of most of the sulphydryl and some nonsulphydryl enzymes $[26,65]$. Lipid peroxidation can be induced by many chemicals like kainic acid, Pc, and tissue injuries, and it has been suggested for a possible mechanism for the neurotoxic effects observed during epileptic activity
$[5,26]$. The present findings clearly indicated that lipid peroxidation levels (TBARS) in the striatum and hippocampus of rats were increased and the reduced glutathione (GSH) concentration was decreased after seizure activities of SE induced by Li-Pc. In normal conditions, there is a steady state balance between the ROS production (TBARS) and their scavenging by the cellular antioxidant system (GSH). Presence of Pc may be associated with marked alterations of enzymatic (SOD, CAT, and GST) and nonenzymatic components (TBARS and GSH) of antioxidant defense system (AOS). The comparative study of the three drugs (Qcn, Pgm, and Ptx) demonstrates that these drugs act as effective antioxidants and effectively protect against Pc-induced lipid peroxidation and ameliorate the negative effect of Pc on antioxidant status by protecting the effects from oxidative stress and have an ameliorating effect on the AOS. Thus it is likely that this pathomechanism may contribute at least in part to the pathophysiology of the seizure activity. Antioxidant therapies have been of great interest in the treatment of neurodegenerative disorders [66]. Among the three drugs studied herein, Ptx is found to have a $100 \%$ survival rate in the SE animals without any mortality and has the highest neuroprotection from SE in the cognitive behavioral and biochemical parameters as observed herein and as reported in an earlier study [6]. Ptx has been reported as a potent free radical scavenger [67] and the neuroprotective effect of Ptx has been attributed to its antioxidant activity by earlier investigators [34, 35]. The present study clearly suggests that Ptx, Qcn, and Pgm are potent free radical scavengers and have antiepileptic as well as antioxidant activity in the order Ptx $>$ Pgm $>$ Qcn. Antioxidants and free radical scavengers have been shown to reduce neuropathology associated with SE [6].

\section{Conclusion}

In conclusion, the present study suggests that all the drugs used in the present study have potential for being antiepileptic as well as antioxidant in an effective order of Ptx $<$ Pgm $<$ Qcn. However, further studies can confirm these effects and also can indicate whether OS plays a definite role in the pathophysiology of installation and/or propagation of epileptic seizures. It is however assumed that Ptx, Pgm, and Qcn combined with conventional therapies might provide a beneficial effect in the treatment of epilepsy through ameliorating the cerebral OS.

\section{Conflict of Interests}

The authors declare that there is no conflict of interests regarding the publication of this paper.

\section{Acknowledgment}

The authors would like to extend their sincere appreciation to the Deanship of Scientific Research at King Saud University, Riyadh, Saudi Arabia, for funding of this research through the Research Group Project no. RGP-VPP-240. 


\section{References}

[1] S. Chen, S. Fujita, N. Koshikawa, and M. Kobayashi, "Pilocarpine-induced status epilepticus causes acute interneuron loss and hyper-excitatory propagation in rat insular cortex," Neuroscience, vol. 166, no. 1, pp. 341-353, 2010.

[2] M. Ahmad, "Protective effects of curcumin against lithiumpilocarpine induced status epilepticus, cognitive dysfunction and oxidative stress in young rats," Saudi Journal of Biological Sciences, vol. 20, no. 2, pp. 155-162, 2013.

[3] E. A. Cavalheiro, M. J. Fernandes, L. Turski, and M. G. NaffahMazzacoratti, "Spontaneous recurrent seizures in rats: amino acid and monoamine determination in the hippocampus," Epilepsia, vol. 35, no. 1, pp. 1-11, 1994.

[4] R. M. de Freitas, F. C. F. de Sousa, S. M. M. Vasconcelos, G. S. B. Viana, and M. M. D. F. Fonteles, "Acute alterations of neurotransmitters levels in striatum of young rat after pilocarpineinduced status epilepticus," Arquivos de Neuro-Psiquiatria, vol. 61, no. 2, pp. 430-433, 2003.

[5] R. M. Freitas, S. M. M. Vasconcelos, F. C. F. Souza, G. S. B. Viana, and M. M. F. Fonteles, "Monoamine levels after pilocarpineinduced status epilepticus in hippocampus and frontal cortex of Wistar rats," Neuroscience Letters, vol. 370, no. 2-3, pp. 196-200, 2004.

[6] M. Tariq, M. Ahmad, K. A. Moutaery, and S. A. Deeb, "Pentoxifylline ameliorates lithium-pilocarpine induced status epilepticus in young rats," Epilepsy and Behavior, vol. 12, no. 3, pp. 354-365, 2008.

[7] A. J. Bruce and M. Baudry, "Oxygen free radicals in rat limbic structures after kainate-induced seizures," Free Radical Biology and Medicine, vol. 18, no. 6, pp. 993-1002, 1995.

[8] R. M. Freitas, S. M. M. Vasconcelos, F. C. F. Souza, G. S. B. Viana, and M. M. F. Fonteles, "Oxidative stress in the hippocampus after pilocarpine-induced status epilepticus in Wistar rats," FEBS Journal, vol. 272, no. 6, pp. 1307-1312, 2005.

[9] M. I. Bellissimo, D. Amado, D. S. P. Abdalla, E. C. Ferreira, E. A. Cavalheiro, and M. Da Graça Naffah-Mazzacoratti, "Superoxide dismutase, glutathione peroxidase activities and the hydroperoxide concentration are modified in the hippocampus of epileptic rats," Epilepsy Research, vol. 46, no. 2, pp. 121-128, 2001.

[10] J. Liu, A. Wang, L. Li, Y. Huang, P. Xue, and A. Hao, "Oxidative stress mediates hippocampal neuron death in rats after lithiumpilocarpine-induced status epilepticus," Seizure, vol. 19, no. 3, pp. 165-172, 2010.

[11] C. Ikonomidou and A. M. Kaindl, "Neuronal death and oxidative stress in the developing brain," Antioxidants and Redox Signaling, vol. 14, no. 8, pp. 1535-1550, 2011.

[12] J. Folbergrová and W. S. Kunz, "Mitochondrial dysfunction in epilepsy," Mitochondrion, vol. 12, no. 1, pp. 35-40, 2012.

[13] S. M. Xavier, C. O. Barbosa, D. O. Barros, R. F. Silva, A. A. Oliveira, and R. M. Freitas, "Vitamin C antioxidant effects in hippocampus of adult Wistar rats after seizures and status epilepticus induced by pilocarpine," Neuroscience Letters, vol. 420, no. 1, pp. 76-79, 2007.

[14] D. O. Barros, S. M. L. Xavier, C. O. Barbosa et al., "Effects of the vitamin $\mathrm{E}$ in catalase activities in hippocampus after status epilepticus induced by pilocarpine in Wistar rats," Neuroscience Letters, vol. 416, no. 3, pp. 227-230, 2007.

[15] R. M. Freitas, "Investigation of oxidative stress involvement in hippocampus in epilepsy model induced by pilocarpine," Neuroscience Letters, vol. 462, no. 3, pp. 225-229, 2009.
[16] H. Shibley and B. N. Smith, "Pilocarpine-induced status epilepticus results in mossy fiber sprouting and spontaneous seizures in C57BL/6 and CD-1 mice," Epilepsy Research, vol. 49, no. 2, pp. 109-120, 2002.

[17] H. S. Aboul Ezz, Y. A. Khadrawy, and N. A. Noor, "The neuroprotective effect of curcumin and nigella sativa oil against oxidative stress in the pilocarpine model of epilepsy: a comparison with valproate," Neurochemical Research, vol. 36, no. 11, pp. 2195-2204, 2011.

[18] P. DU, H.-Y. Tang, X. Li et al., "Anticonvulsive and antioxidant effects of curcumin on pilocarpine-induced seizures in rats," Chinese Medical Journal, vol. 125, no. 11, pp. 1975-1979, 2012.

[19] K. H. Reeta, J. Mehla, and Y. K. Gupta, "Curcumin is protective against phenytoin-induced cognitive impairment and oxidative stress in rats," Brain Research, vol. 1301, pp. 52-60, 2009.

[20] K. H. Reeta, J. Mehla, and Y. K. Gupta, "Curcumin ameliorates cognitive dysfunction and oxidative damage in phenobarbitone and carbamazepine administered rats," European Journal of Pharmacology, vol. 644, no. 1-3, pp. 106-112, 2010.

[21] K. H. Reeta, J. Mehla, M. Pahuja, and Y. K. Gupta, "Pharmacokinetic and pharmacodynamic interactions of valproate, phenytoin, phenobarbitone and carbamazepine with curcumin in experimental models of epilepsy in rats," Pharmacology Biochemistry and Behavior, vol. 99, no. 3, pp. 399-407, 2011.

[22] A. Ataie, M. Sabetkasaei, A. Haghparast, A. H. Moghaddam, and B. Kazeminejad, "Neuroprotective effects of the polyphenolic antioxidant agent, curcumin, against homocysteine-induced cognitive impairment and oxidative stress in the rat," Pharmacology Biochemistry and Behavior, vol. 96, no. 4, pp. 378-385, 2010.

[23] Y. K. Gupta and S. Briyal, "Protective effect of vineatrol against kainic acid induced seizures, oxidative stress and on the expression of heat shock proteins in rats," European Neuropsychopharmacology, vol. 16, no. 2, pp. 85-91, 2006.

[24] Y. K. Gupta, S. Briyal, and M. Sharma, "Protective effect of curcumin against kainic acid induced seizures and oxidative stress in rats," Indian Journal of Physiology and Pharmacology, vol. 53, no. 1, pp. 39-46, 2009.

[25] R. L. M. de Freitas, Í. M. Santos, G. F. de Souza, A. D. R. Tomé, G. B. Saldanha, and R. M. de Freitas, "Oxidative stress in rat hippocampus caused by pilocarpine-induced seizures is reversed by buspirone," Brain Research Bulletin, vol. 81, no. 4-5, pp. 505-509, 2010.

[26] J. C. Gilbert and A. H. Sawas, "ATPase activities and lipid peroxidation in rat cerebral cortex synaptosomes," Archives Internationales de Pharmacodynamie et de Therapie, vol. 263, no. 2, pp. 189-196, 1983.

[27] N. Rathor, T. Arora, S. Manocha, A. N. Patil, P. K. Mediratta, and K. K. Sharma, "Anticonvulsant activity of Aloe vera leaf extract in acute and chronic models of epilepsy in mice," Journal of Pharmacy and Pharmacology, vol. 66, no. 3, pp. 477-485, 2014.

[28] R.-R. Zhao, X.-C. Xu, F. Xu et al., "Metformin protects against seizures, learning and memory impairments and oxidative damage induced by pentylenetetrazole-induced kindling in mice," Biochemical and Biophysical Research Communications, vol. 448, no. 4, pp. 414-417, 2014.

[29] B. S. Bunney, L. A. Chiodo, and A. S. Freeman, "Further studies on the specificity of proglumide as a selective cholecystokinin antagonist in the central nervous system," Annals of the New York Academy of Sciences, vol. 448, pp. 345-351, 1985.

[30] C. Schwarzer, J. M. Williamson, E. W. Lothman, A. Vezzani, and G. Sperk, "Somatostatin, neuropeptide Y, neurokinin B and 
cholecystokinin immunoreactivity in two chronic models of temporal lobe epilepsy," Neuroscience, vol. 69, no. 3, pp. 831-845, 1995.

[31] C. Vigo, G. P. Lewis, and P. J. Piper, "Mechanisms of inhibition of phospholipase A2," Biochemical Pharmacology, vol. 29, no. 4, pp. 623-627, 1980.

[32] J. W. Phillis, "Cerebroprotective action of the phospholipase inhibitor quinacrine in the ischemia/reperfused gerbil, hippocampus," Life Sciences, vol. 58, no. 6, pp. PL97-PL101, 1996.

[33] G. M. A. Cunha, P. J. P. Bezerra, M. D. D. Saldanha, M. C. Cavalcante, M. S. D. de Brun, and G. S. B. Viana, "Pentoxifylline improves learning and memory in glutamate-lesioned rats," Pharmacology Biochemistry and Behavior, vol. 6, pp. 687-694, 2000.

[34] S. Savaş, N. Delibaş, Ç. Savaş, R. Sütçü, and A. Cindaş, "Pentoxifylline reduces biochemical markers of ischemia-reperfusion induced spinal cord injury in rabbits," Spinal Cord, vol. 40, no. 5, pp. 224-229, 2002.

[35] C. Banfi, L. Sironi, G. de Simoni et al., "Pentoxifylline prevents spontaneous brain ischemia in stroke-prone rats," Journal of Pharmacology and Experimental Therapeutics, vol. 310, no. 3, pp. 890-895, 2004.

[36] F. Dal-Pizzol, F. Klamt, M. M. R. Vianna et al., "Lipid peroxidation in hippocampus early and late after status epilepticus induced by pilocarpine or kainic acid in Wistar rats," Neuroscience Letters, vol. 291, no. 3, pp. 179-182, 2000.

[37] W. A. Turski, "Pilocarpine-induced seizures in rodents-17 years on," Polish Journal of Pharmacology, vol. 52, no. 1, pp. 6365, 2000.

[38] R. Druga, H. Kubová, L. Suchomelová, and R. Haugvicová, "Lithium/pilocarpine status epilepticus-induced neuropathology of piriform cortex and adjoining structures in rats is agedependent," Physiological Research, vol. 52, no. 2, pp. 251-264, 2003.

[39] H. Kubová, P. Mareš, L. Suchomelová, G. Brožek, R. Druga, and A. Pitkänen, "Status epilepticus in immature rats leads to behavioural and cognitive impairment and epileptogenesis," European Journal of Neuroscience, vol. 19, no. 12, pp. 3255-3265, 2004.

[40] L. Turski, C. Ikonomidou, W. A. Turski, Z. A. Bortolotto, and E. A. Cavalheiro, "Review: cholinergic mechanisms and epileptogenesis. The seizures induced by pilocarpine: a novel experimental model of intractable epilepsy," Synapse, vol. 3, no. 2, pp. 154-171, 1989.

[41] C. Dubé, S. Boyet, C. Marescaux, and A. Nehlig, "Relationship between neuronal loss and interictal glucose metabolism during the chronic phase of the lithium-pilocarpine model of epilepsy in the immature and adult rat," Experimental Neurology, vol. 167, no. 2, pp. 227-241, 2001.

[42] M. A. Persinger, Y. R. J. Bureau, M. Kostakos, O. Peredery, and H. Falter, "Behaviors of rats with insidious, multifocal brain damage induced by seizures following single peripheral injections of lithium and pilocarpine," Physiology and Behavior, vol. 53, no. 5, pp. 849-866, 1993.

[43] S. Faverjon, D. C. Silveira, D. D. Fu et al., "Beneficial effects of enriched environment following status epilepticus in immature rats," Neurology, vol. 59, no. 9, pp. 1356-1364, 2002.

[44] A. Rutten, M. Van Albada, D. C. Silveira et al., "Memory impairment following status epilepticus in immature rats: time-course and environmental effects," European Journal of Neuroscience, vol. 16, no. 3, pp. 501-513, 2002.
[45] R. Morris, "Developments of a water-maze procedure for studying spatial learning in the rat," Journal of Neuroscience Methods, vol. 11, no. 1, pp. 47-60, 1984.

[46] H. J. Spiers, N. Burgess, T. Hartley, F. Vargha-Khadem, and J. O'Keefe, "Bilateral hippocampal pathology impairs topographical and episodic memory but not visual pattern matching," Hippocampus, vol. 11, no. 6, pp. 715-725, 2001.

[47] V. S. Nascimento, A. A. Oliveira, R. M. Freitas et al., "Pilocarpine-induced status epilepticus: monoamine level, muscarinic and dopaminergic receptors alterations in striatum of young rats," Neuroscience Letters, vol. 383, no. 1-2, pp. 165-170, 2005.

[48] R. M. Freitas, A. D. A. Oliveira, S. M. M. Vasconcelos, F. C. F. Sousa, G. S. B. Viana, and M. M. F. Fonteles, "Expression of muscarinic and dopaminergic receptors and monoamine levels frontal cortex of epileptic rats," Pharmacology Biochemistry and Behavior, vol. 83, no. 2, pp. 302-306, 2006.

[49] H. Ohkawa, N. Ohishi, and K. Yagi, "Assay for lipid peroxides in animal tissues by thiobarbituric acid reaction," Analytical Biochemistry, vol. 95, no. 2, pp. 351-358, 1979.

[50] M. J. Mangino, M. K. Murphy, G. G. Grabau, and C. B. Anderson, "Protective effects of glycine during hypothermic renal ischemia-reperfusion injury," The American Journal of Physiology-Renal Fluid and Electrolyte Physiology, vol. 261, no. 5, pp. F841-F848, 1991.

[51] W. H. Habig, M. J. Pabst, and W. B. Jakoby, "Glutathione S-transferases. The first enzymatic step in mercapturic acid formation," The Journal of Biological Chemistry, vol. 249, no. 22, pp. 7130-7139, 1974.

[52] H. Aebi, "Catalase," in Methods of Enzymatic Analysis, H. U. Bergmeter, Ed., vol. 2, Academic Press, New York, NY, USA, 1972.

[53] H. P. Misra and I. Fridovich, "The role of superoxide anion in the autoxidation of epinephrine and a simple assay for superoxide dismutase," The Journal of Biological Chemistry, vol. 247, no. 10, pp. 3170-3175, 1972.

[54] Z. Liu, C. E. Stafstrom, M. Sarkisian et al., "Age-dependent effects of glutamate toxicity in the hippocampus," Developmental Brain Research, vol. 97, no. 2, pp. 178-184, 1996.

[55] C.-L. Wu, L.-T. Huang, C.-W. Liou et al., "Lithium-pilocarpineinduced status epilepticus in immature rats result in longterm deficits in spatial learning and hippocampal cell loss," Neuroscience Letters, vol. 312, no. 2, pp. 113-117, 2001.

[56] B. H. Cha, D. C. Silveira, X. Liu, Y. Hu, and G. L. Holmes, "Effect of topiramate following recurrent and prolonged seizures during early development," Epilepsy Research, vol. 51, no. 3, pp. 217232, 2002.

[57] P. L. Hung, M. C. Lai, S. N. Yang et al., "Aminophylline exacerbates status epilepticus-induced neuronal damages in immature rats: a morphological, motor and behavioral study," Epilepsy Research, vol. 49, no. 3, pp. 218-225, 2002.

[58] R. G. M. Morris, E. I. Moser, G. Riedel et al., "Elements of a neurobiological theory of the hippocampus: the role of activity-dependent synaptic plasticity in memory," Philosophical Transactions of the Royal Society B: Biological Sciences, vol. 358, no. 1432, pp. 773-786, 2003.

[59] M. Higashima, K. Ohno, H. Kinoshita, and Y. Koshino, "Involvement of $\mathrm{GABA}(\mathrm{A})$ and $\mathrm{GABA}(\mathrm{B})$ receptors in afterdischarge generation in rat hippocampal slices," Brain Research, vol. 865, no. 2, pp. 186-193, 2000.

[60] J. E. Walker, E. Lewin, J. R. Sheppard, and R. Cromwell, "Enzymatic regulation of adenosine $3^{\prime}, 5^{\prime}$ monophosphate (cyclic 
AMP) in the freezing epileptogenic lesion of rat brain and in homologous contralateral cortex," Journal of Neurochemistry, vol. 21, no. 1, pp. 79-85, 1973.

[61] G. M. Rose, A. Hopper, M. de Vivo, and A. Tehim, "Phosphodiesterase inhibitors for cognitive enhancement," Current Pharmaceutical Design, vol. 11, no. 26, pp. 3329-3334, 2005.

[62] M. M. F. Marinho, F. C. F. de Sousa, V. M. S. de Bruin, M. R. Vale, and G. S. B. Viana, "Effects of lithium, alone or associated with pilocarpine, on muscarinic and dopaminergic receptors and on phosphoinositide metabolism in rat hippocampus and striatum," Neurochemistry International, vol. 33, no. 4, pp. 299306, 1998.

[63] M. Patel, "Mitochondrial dysfunction and oxidative stress: cause and consequence of epileptic seizures," Free Radical Biology and Medicine, vol. 37, no. 12, pp. 1951-1962, 2004.

[64] M. T. Lin and M. F. Beal, "Mitochondrial dysfunction and oxidative stress in neurodegenerative diseases," Nature, vol. 443, no. 7113, pp. 787-795, 2006.

[65] M. Hayashi, "Oxidative stress in developmental brain disorders," Neuropathology, vol. 29, no. 1, pp. 1-8, 2009.

[66] T. R. Golden and M. Patel, "Catalytic antioxidants and neurodegeneration," Antioxidants and Redox Signaling, vol. 11, no. 3, pp. 555-569, 2009.

[67] V. B. Bhat and K. M. Madyastha, "Antioxidant and radical scavenging properties of 8-oxo derivatives of xanthine drugs pentoxifylline and lisofylline," Biochemical and Biophysical Research Communications, vol. 288, no. 5, pp. 1212-1217, 2001. 


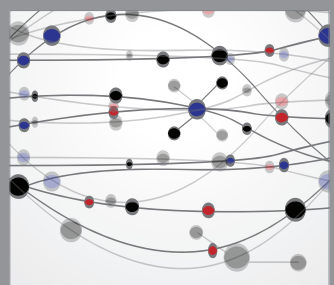

The Scientific World Journal
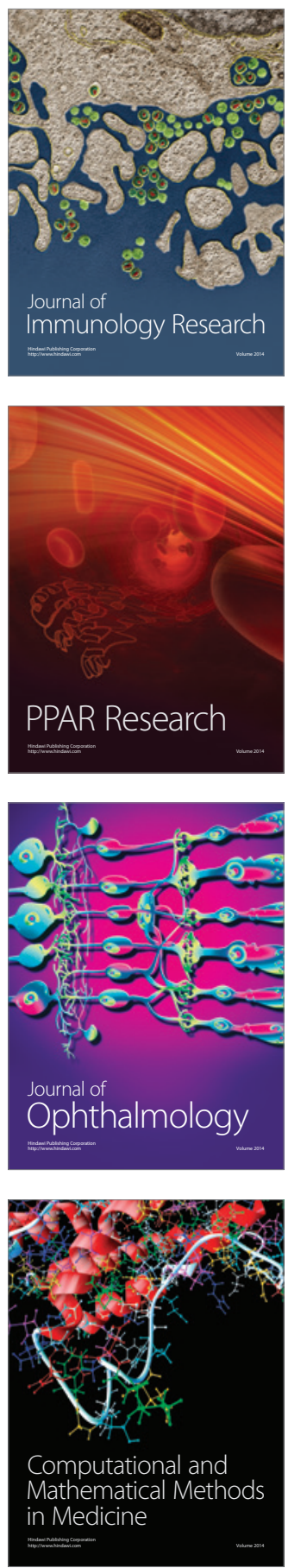

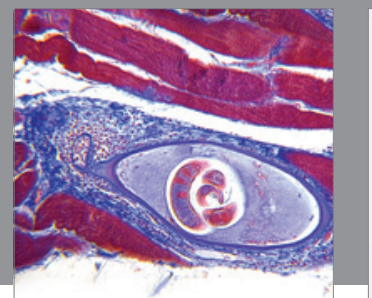

Gastroenterology

Research and Practice
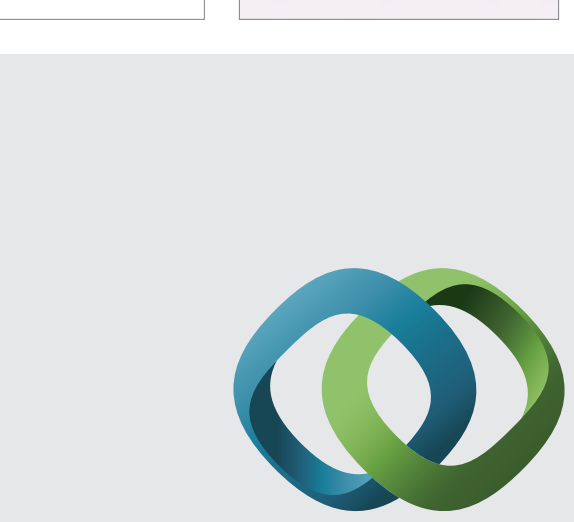

\section{Hindawi}

Submit your manuscripts at

http://www.hindawi.com
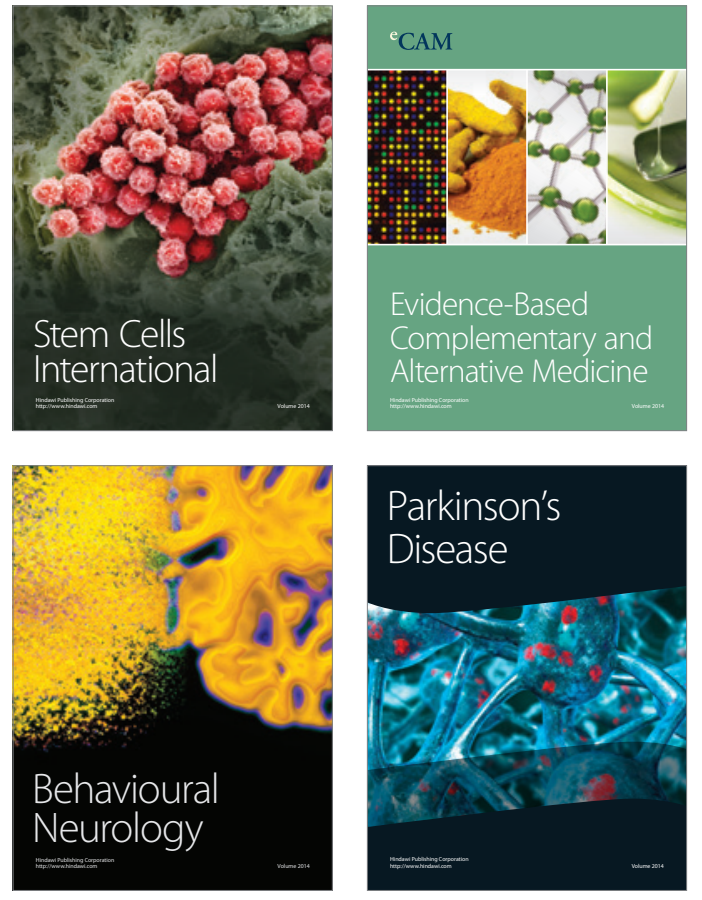
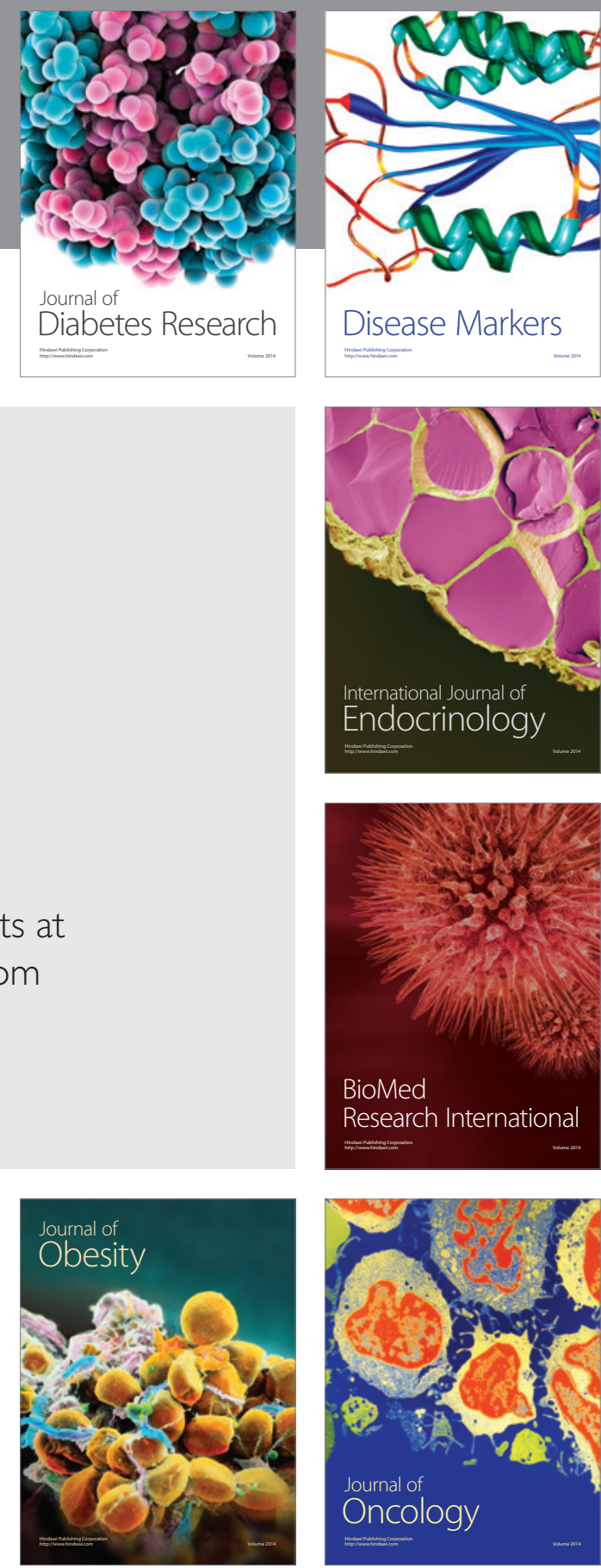

Disease Markers
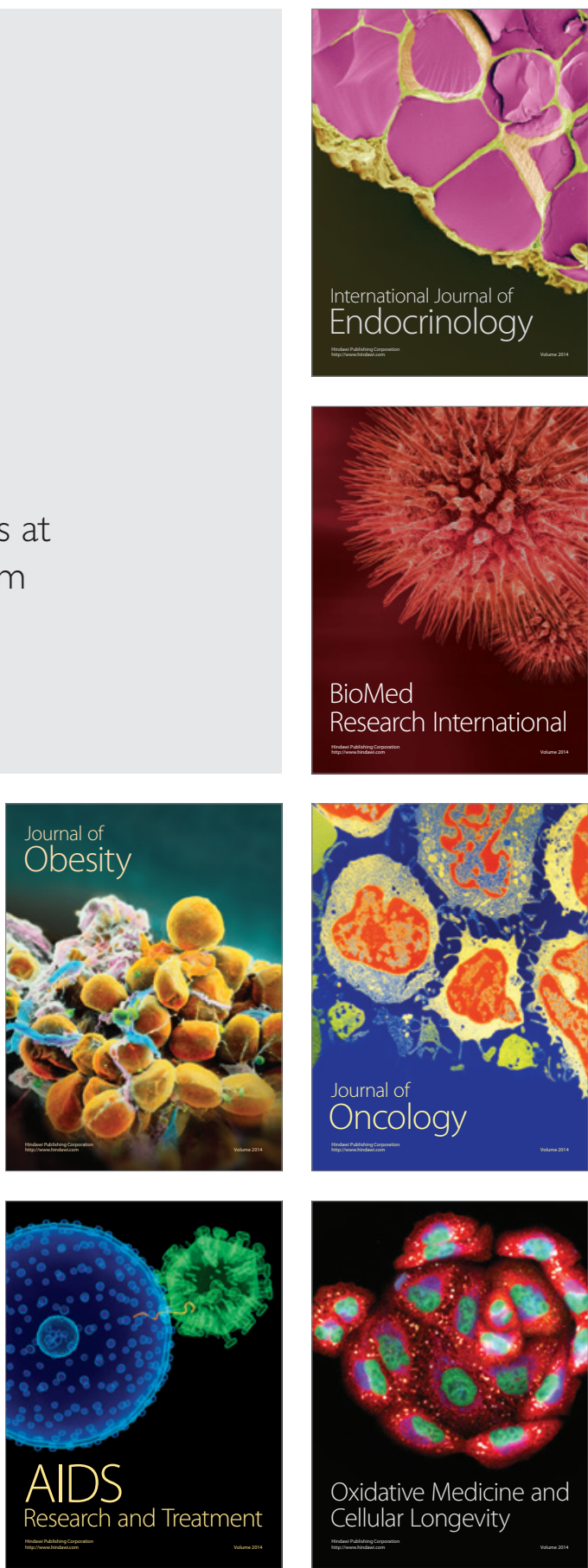